\title{
Huella de Carbono, un Concepto que no puede estar Ausente en Cursos de Ingeniería y Ciencias
}

\author{
José O. Valderrama ${ }^{(1,2)}$, César Espíndola $\left(^{3,4)}\right.$ y Rafael Quezada ${ }^{3}$ \\ (1) Univ. de La Serena, Fac. de Ingeniería, Dpto. de Ing. Mecánica., Casilla 554, La Serena-Chile \\ (2) Centro de Información Tecnológica (CIT), Casilla 724, La Serena-Chile \\ 3) Univ. de La Serena, Fac. de Ingeniería, Dpto. de Ing. Industrial, Casilla 554, La Serena-Chile \\ (4) Univ. de Lleida, Depto. Adm. Empresas y Gestión Econ. de Rec. Naturales, Lleida-España
}

Recibido Ene. 30, 2011; Aceptado Feb. 25, 2011; Versión final recibida Mar. 14, 2011

\section{Resumen}

Se analiza el concepto de huella del carbono ( $\mathrm{HdC})$, se discute su relación con la emisión de gases contaminantes, y se justifica su inclusión en cursos formales de ingeniería y ciencias. Los avances logrados en este tema por organizaciones internacionales y empresas en el mundo hacen atractivo y necesario discutir el tema de la $\mathrm{HdC}$ en cursos universitarios. Actualmente el tema está confinado a algunos cursos electivos y a carreras del ámbito ambiental. Se proponen formas de motivar e incentivar a los alumnos a través de trabajo de investigación, presentaciones orales e informes escritos. Se dan ejemplos de temas que pueden ser abordados en un curso universitario o de nivel técnico superior. Se concluye que por tratarse de una materia transversal que afecta a todos los sectores, los futuros profesionales de las ingenierías y las ciencias deben tener las bases mínimas que les permitan entender el concepto y aplicarlo durante su vida profesional.

\section{Carbon Footprint, a Concept that cannot be Absent in Courses of Engineering and Sciences}

\begin{abstract}
The concept of Carbon Footprint is analyzed, its relation to emission of dangerous gases is discussed, and its inclusion in formal undergraduate courses of engineering and sciences is explained. The advances done on this subject by international organizations and companies around the world makes it attractive and necessary to discuss the subject of carbon footprint in university courses. At present, this matter is only treated in elective courses and in some programs of environmental engineering. The paper proposes some forms for motivating students through semester research projects, oral presentations, and written essays. Examples of subjects that can be assigned in undergraduate or graduate courses are provided. It is concluded that being this a wide subject that touches all sectors, the future engineers and scientists must have the minimum knowledge that allows them to understand the concept and apply it in their professional life.
\end{abstract}

Keywords: carbon footprint, teaching-learning, professional formation, greenhouse gases 


\section{INTRODUCCIÓN}

La mayor parte de la comunidad científica y un número creciente de grupos sociales, empresariales y políticos de los más diversos países han aceptado las evidencias de que el cambio climático es originado por las actividades humanas, llegando a la conclusión de que éste constituye uno de los mayores desafíos ambientales que se pudiera interponer en el camino hacia el desarrollo sustentable en el presente siglo (WRI, 2008). Así también, es ampliamente aceptado que la causa de dicho fenómeno se encontraría en las altas concentraciones atmosféricas de Gases Efecto Invernaderos (GEI), las cuales serían responsables del aumento de la temperatura global del planeta (IPCC, 2007). El Panel Intergubernamental sobre Cambio Climático con sede en Ginebra-Suiza, ha indicado que el riesgo del cambio climático es severo y que su impacto aumentará notablemente con un incremento de las temperaturas en $2{ }^{\circ} \mathrm{C}$ por encima de las registradas en la época preindustrial (EPA, 2006).

El cambio climático no sólo constituye un problema ambiental sino, también, un problema de desarrollo, con profundos impactos potenciales en la sociedad, la economía y los ecosistemas. El debate sobre el cambio climático ha trascendido al comercio internacional y, es liderado por los países con compromisos de reducción de emisiones. Este especial interés es motivado fundamentalmente por la preocupación de los países comprometidos por las posibles pérdidas de competitividad de sus productores, quienes estarían compitiendo con otros exportadores con menores costos de emisión que no han asumido obligaciones climáticas (De la Torre et al., 2009). En el caso de los países en vías de desarrollo, como lo son la mayoría de los países latinoamericanos, un patrón exportador más acorde con las aspiraciones del desarrollo sostenible y menos vulnerable a las exigencias climáticas de un paradigma económico carbono reduccionista, exige a los sectores productivos a avanzar rápidamente en los proceso de cuantificación y de disminución de los efectos climáticos, con el fin de resguardar su actual posición competitiva.(Schneider y Samaniego, 2009; Clément y Lenne, 2010).

Esta situación ha provocado la aparición de nuevos temas en la agenda comercial, siendo la Huella de Carbono (HC) la que ha cobrado una mayor relevancia. La HC, comúnmente definida como la cantidad de gases efecto invernadero emitidos a la atmósfera, derivados de las actividades de producción o consumo de bienes y servicios, y se ha transformado en un patrón de competitividad. (Wiedmann y Minx, 2008). Este indicador ha provocado que otros temas relacionados con las emisiones, tales como la fuga del carbono, el análisis del ciclo de vida en la cadena productiva y comercial, las responsabilidades en torno a la contabilidad global del carbono adquieran relevancia en el ámbito internacional. Esta importancia se ve reflejada en las presiones de los países comprometidos con el tema ambiental, para que aquellos en vías de desarrollo asuman compromisos comparables. Este proceso ha motivado la implementación de medidas tales como los impuestos al carbono, los programas de transacción de derechos de emisión, las barreras técnicas, las exigencias de eficiencia energética y el control de emisiones de GEI (Clément y Lenne, 2010).

Esta situación ha llevado también a diferentes organizaciones públicas, privadas y por cierto al mundo académico, a discutir y proponer metodologías y regulaciones para contabilizar y declarar impactos ambientales de la producción y/o el consumo de bienes y servicios, así como las emisiones corporativas de los GEl, a través del uso de la HdC. Sin embargo, la HdC, a pesar de contar un origen conocido en la Huella Ecológica de Wackernagel y Rees (1995), tiene hoy en día muy diversas interpretaciones. Esto ha llevado al desarrollo de metodologías de cálculo muy disímiles, situación que genera una excesiva controversia frente a un índice que goza de bastante popularidad (Carballo y García, 2008).

A pesar de la importancia actual y su potencial creciente influencia futura, el concepto de HdC no ha sido incorporado en forma adecuada en cursos relacionados con el medio ambiente o en cursos de gestión donde es necesario aplicar y cuantificar este concepto. Existen iniciativas en algunos países desarrollados en los que este concepto es ya incorporado en la escuela secundaria y existen una gran cantidad de investigación de posgrado alrededor del mundo (SchoolCoop, 2011; NEA 2011). En la investigación académica el tema ha experimentado también 
un notable desarrollo. Desde unas pocas publicaciones a fines del siglo pasado la literatura relacionada con la $\mathrm{HdC}$ ha experimentado un crecimiento exponencial en los últimos 3 años. La Fig. 1 muestra el número de publicaciones encontradas en la literatura internacional relacionadas con la huella del carbono y temas afines en los últimos 15 años.

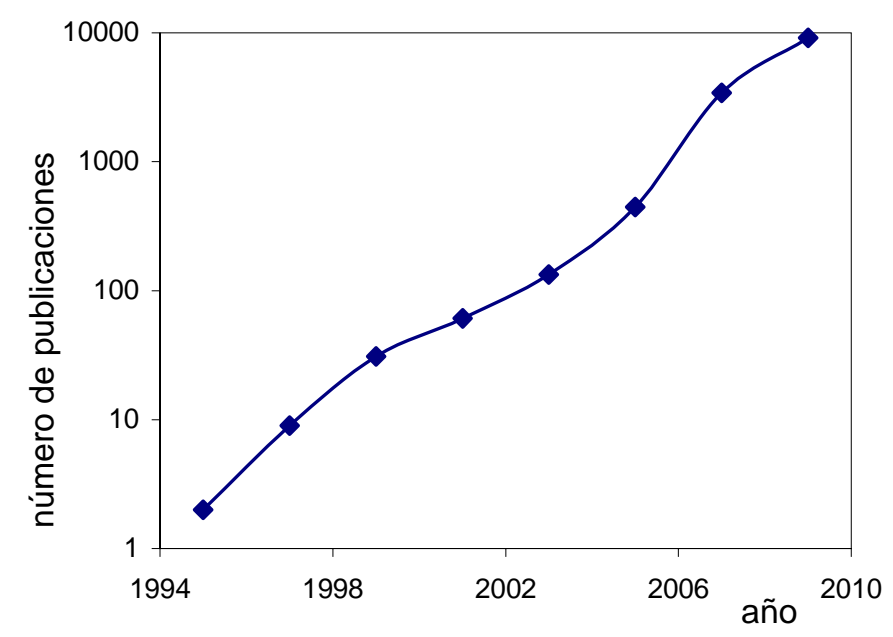

Fig. 1: Publicaciones en la literatura internacional relacionados con la huella del carbono

Sin embargo, como se trata de un tema transversal que afecta a todos los sectores los futuros profesionales deben tener las bases mínimas que les permitan entender el concepto y aplicarlo durante su vida profesional. En este artículo se presentan algunos aspectos generales sobre la $\mathrm{HdC}$, de modo que profesores involucrados en cursos relacionados con medio ambiente, con contaminación ambiental, con tratamiento de residuos y con tópicos afines, puedan incorporarlo como tema y materia de discusión. Por tratarse de un tema en que no hay demasiados aspectos teóricos, este trabajo enfatiza la enseñanza de los conceptos fundamentales y de sus aplicaciones a través de trabajos semestrales de investigación y desarrollo.

\section{LA DEFINICIÓN DE LA HdC Y SU ORIGEN}

Todos los productos que se consumen y los servicios que se prestan tienen un impacto sobre el clima y producen gases de efecto invernadero durante su producción, transporte, almacenamiento, uso y disposición final. La HdC ha surgido como una medida de la cuantificación del efecto de estos GEI. En este contexto, la HdC se ha convertido en un lema en el debate público sobre el cambio climático, atrayendo la atención de los consumidores, negocios, gobiernos, organizaciones no-gubernamentales, e instituciones internacionales (Peters y Hertwich, 2008). A pesar de su amplio uso, la literatura reconoce la ausencia de una clara definición que sea comúnmente aceptada (Wiedmann y Minx, 2008). La HdC, sólo ha surgido en el dominio público en los últimos años como una descripción bastante general de la emisión de gases de efecto invernadero totales asociados con la actividad humana. Impulsado por un uso generalizado en los medios de comunicación y el público en general, la $\mathrm{HdC}$ se ha convertido en un sinónimo de los efectos del cambio climático de los individuos, comunidades, naciones, empresas o productos. Según Wiedmann y Minx, (2008).el mundo académico debe ponerse al día con este despliegue rápido de una expresión de la cual todavía se está debatiendo una definición precisa.

Dado el reciente interés en el concepto de $\mathrm{HdC}$, no es sorprendente que muchas personas piensen que el concepto $\mathrm{HdC}$ es algo nuevo. Sin embargo, se encuentran antecedentes de los marcos metodológicos utilizados para cálculo de HdC desde hace ya bastante tiempo (Minx et al., 2010). Desde fines de los años 80 del siglo pasado se encuentran aplicaciones de la HdC en la literatura, aunque bajo nombres diferentes (Minx et al., 2010). El debate sobre la HdC no sólo alcanza a su origen sino también al enfoque metodológico empleado en su análisis. Uno de los cuestionamientos más fuertes es que huella debe, por su naturaleza, abarcar todas las huellas 
que deja tras de sí una actividad. En el caso de la HdC se debe incluir todas las emisiones de gases de efecto invernadero que se pueden asociar directa e indirectamente con una actividad, proceso o elaboración de productos y servicios.

Frente a la atención internacional sin precedentes que ha captado en los últimos años, el problema del cambio climático la huella de carbono surge, entonces, como un indicador capaz de sintetizar en forma más o menos fidedigna los impactos provocados por las actividades del hombre en el entorno, medido en términos de emisiones de GEI y se perfila como una herramienta eficaz de gestión empresarial (Wittneben y Kiyar, 2009). El uso de la HdC ha encontrado un importante campo de aplicación como herramienta para cuantificar la eficiencia energética y su impacto en los costos operacionales de la empresa, situación que puede mejorar el margen de beneficios de la empresa contribuyendo no sólo a la sustentabilidad ambiental sino también a la rentabilidad económica de la misma. Sin embargo, los tomadores de decisiones deben ser conscientes de la incertidumbre que rodea a todo cálculo de la HdC. De lo contrario, pueden llegar a interpretaciones incorrectas de los resultados y a conclusiones erróneas (Plasmann et al., 2010). A pesar de estas incertidumbres, la HdC se perfila como un indicador capaz de sintetizar los impactos provocados por las actividades del hombre en el entorno, medido en términos de emisiones de GEI y se presenta como una poderosa herramienta de gestión y un estímulo para adoptar una estrategia proactiva en el logro de la sustentabilidad de las organizaciones. (Wiedmann y Minx, 2008; Boiral, 2006; Wittneben y Kiyar, 2009).

Si existieran estándares comparables, que no los hay aún, la HdC podría ayudar a una empresa en varios frentes: i) Crear transparencia en la cadena de valor en relación a los procesos y los actores involucrados; ii) Aumentar la conciencia sobre la emisión de gases de efecto invernadero e identificar procesos particulares de emisiones extremas identificando a la persona que es responsable del mismo; iii) Identificar áreas donde hay potencial para reducir emisiones, y optimizar operaciones y costos; iv) Contar con una herramienta eficaz para la gestión energética y ambiental de la compañía, mediante el adecuado manejo de sus emisiones de CO2e; v) Poseer una base de información para anticiparse a futuras regulaciones; vi) Analizar y evaluar que tan relevantes son las emisiones de gases de efecto invernadero en comparación a otros impactos que tiene el producto en el medio ambiente; y vii) Fortalecer la posición competitiva a través de la comunicación, por medio de etiquetados o de informes de sustentabilidad corporativa, dirigida .a los grupos de interés: empleados, clientes, proveedores, accionistas, bancos, ambientalistas, gobierno u otros grupos que puedan ayudar o dañar a la corporación.

\section{CRITERIOS DE COMPARACIÓN DE LOS CÁLCULOS DE HdC}

Existen cuatro métodos principales presentados en la literatura para el cálculo de la HdC en empresas, organizaciones, servicios, procesos y productos. Estos métodos utilizan un acercamiento muy similar para la obtención de datos como es el análisis de ciclo de vida del producto. Una vez que se esquematiza el ciclo de vida del producto, desde que se extraen las materias primas necesarias para su fabricación hasta el lugar de su disposición final, cada método usa un enfoque diferente. La primera diferencia tiene lugar en la limitación del alcance de la empresa, es decir hasta dónde las emisiones de otros procesos influyen en el proceso en estudio. Una vez que se tiene el proceso bien delimitado, se extraen datos diferentes del proceso, para cada método de determinación. La Tabla 1 resume estos métodos.

El Protocolo de Gases Efecto Invernadero (Protocolo GEl), fue implementado en el 2001 por el Consejo Mundial de Negocios por el Desarrollo Sustentable (World Business Council for Sustainable Development, WBCSD) y por el Instituto de Recursos Mundiales (World Resources Institute, WRI) que tiene como meta el establecimiento de bases para la contabilización de emisiones de los GEI. Es fruto de una colaboración multilateral entre empresas, organizaciones no-gubernamentales y gobiernos. Esta iniciativa cuenta con el apoyo de la Agencia para el Desarrollo Internacional de los Estados Unidos (United States Agency for International Development, USAID) y la Agencia de Protección Ambiental de los Estados Unidos (United States Environmental Protection Agency, USEPA). El Protocolo GEI es un marco metodológico general que da pautas de trabajo para el desarrollo de herramientas de cálculo de emisiones (software). 
Tabla 1. Métodos más relevantes para la determinación de la huella del carbono

\begin{tabular}{|l|l|l|l|l|l|}
\hline Metodologias & Enfoque & Escala & Unidades & Actividades & Referencia \\
\hline $\begin{array}{l}\text { Protocolo de Gases } \\
\text { Efecto Invernadero }\end{array}$ & Corporativo & Empresa & $\mathrm{kg} \mathrm{CO2} \mathrm{eq/año}$ & $\begin{array}{l}\text { todas las } \\
\text { actividades de } \\
\text { una organización }\end{array}$ & $\begin{array}{l}\text { Protocolo GEI, } \\
2005\end{array}$ \\
\hline Balance de Carbono & Corporativo & $\begin{array}{l}\text { Empresa, } \\
\text { Territorio y } \\
\text { Producto: }\end{array}$ & $\begin{array}{l}\mathrm{kg} \mathrm{CO2} \mathrm{eq/año} \\
\mathrm{kg} \mathrm{Co2} \mathrm{eq/Unidad} \\
\text { funcional }\end{array}$ & $\begin{array}{l}\text { todas las } \\
\text { actividades de } \\
\text { una organización }\end{array}$ & $\mathrm{BC,2011}$ \\
\hline $\begin{array}{l}\text { Especificaciones } \\
\text { Públicamente } \\
\text { Disponibles PAS 2050 }\end{array}$ & Producto & Producto: & $\begin{array}{l}\mathrm{kg} \mathrm{CO2} \mathrm{eq/Unidad} \\
\text { funcional }\end{array}$ & $\begin{array}{l}\text { todas las } \\
\text { actividades } \\
\text { productivas }\end{array}$ & $\mathrm{BSI,2008}$ \\
\hline $\begin{array}{l}\text { Método Compuesto } \\
\text { de las Cuentas } \\
\text { Contables MC3 }\end{array}$ & $\begin{array}{l}\text { Corporativo+ } \\
\text { Producto }\end{array}$ & $\begin{array}{l}\text { Empresa: y } \\
\text { Producto: }\end{array}$ & $\begin{array}{l}\text { tco2 eq/año } \\
\text { ton CO2 eq/ton prod. } \\
\text { Ha Globales (Gha) }\end{array}$ & $\begin{array}{l}\text { todas las } \\
\text { actividades de } \\
\text { una organización }\end{array}$ & $\begin{array}{l}\text { Doménech, } \\
2004 a ; ~ 2004 b\end{array}$ \\
\hline
\end{tabular}

La metodología Balance de Carbono, conocida internacionalmente como Bilan Carbone (BC, 2011), fue desarrollada por la Agencia del Medio Ambiente y Energía de Francia (ADEME), en el año 2002. La herramienta fue elaborada específicamente para convertir datos relativos a las actividades productivas en emisiones, de forma rápida, usando factores de emisión (por ejemplo, el consumo de energía de diversos tipos, la cantidad de camiones y la distancia recorrida, el consumo de agua, entre otras). El Bilan Carbone se caracteriza por una visión generalista muy completa, por lo que, a través de sus distintos módulos, permite trabajar a nivel de empresas y eventos pero también de territorios y productos.

El método de las Especificaciones Públicamente Disponibles (Publicly Available Specification), llamado PAS 2050, fue elaborado en el año 2007 por el Instituto Británico de Estandarización (BSI, 2008), con el apoyo del Consorcio del Carbono (Carbon Trust) y el Depto. para el Ambiente, la Alimentación y Asuntos Rurales (DEFRA), ambos organismos del gobierno inglés. El método está enfocado al cálculo de las emisiones de productos y servicios y responde a las normativas ISO y a las del Protocolo GEI. La metodología PAS 2050 define inicialmente las fuentes de emisiones consideradas, además de cinco grandes bloques de actividades, cuyas emisiones deben ser consideradas en la estimación del ciclo de vida de bienes y servicios. Con la enumeración de las fuentes de emisiones consideradas, se realiza una acotación general del ámbito al que se aplica el indicador.

El Método Compuesto de las Cuentas Contables (MC3, Método de las 3 C) fue propuesto por Doménech (2004a) y desarrollado desde entonces por su grupo de investigación. La información necesaria para estimar la $\mathrm{HdC}$ es obtenida principalmente, de documentos contables como el balance y la cuenta de pérdidas y ganancias, lo que permite una clara delimitación de las actividades que están asociadas a cada organización. EI MC3 estima la huella de todos los bienes y servicios recogidos en las cuentas contables, los residuos generados debido a la adquisición de estos bienes y el espacio ocupado por todas las instalaciones de la empresa que recogen las cuentas contables. De esta forma el método MC3 determina la HdC corporativa y la asocia a los productos.

Puesto de una manera simple la $\mathrm{HdC}$ en cualquiera de los métodos descritos arriba es calculada adicionando mediante algún modelo matemático los varios factores que inciden en la producción de GEI. Un modelo simple, para explicar la forma de cálculo, es la suma ponderada de los diversos factores, como se describe en la ecuación. (1)

$H C=\sum x_{i} y_{i} F_{i}$

En esta ecuación general, $x_{i}$ e $y_{i}$ son factores de conversión y equivalencia que dependerán del tipo aplicación (HdC del producto, de una empresa, de un servicio, de una vivienda) y magnitud (local, regional, o de país) y de la influencia de la variable $F_{i}$ (transporte en avión, o auto, uso de carbón o gas natural, emisiones directas o indirectas, kilómetros recorridos, entre muchos otros). 
En una aplicación domestica, por ejemplo en el cálculo de la $\mathrm{HdC}$ anual de una familia, el factor $\mathrm{F}_{1}$ puede ser la electricidad usada, $F_{2}$ la cantidad de autos que tiene la familia o los kilómetros recorridos, $F_{3}$ los metros cuadrados construidos, $F_{4}$ el gasto en supermercado, $F_{5}$ los viajes realizados y las distancias recorridas en avión, entre varios otros factores. En el cálculo de la HdC de un producto, el factor $F_{1}$ puede ser por ejemplo el tipo y tamaño de envase, $F_{2}$ el origen de las materias primas, $F_{3}$ tipo de producto analizado, $F_{4}$ el tipo de proceso al que fue sometido, $F_{5}$ el tipo de energía utilizada en su manufactura, entre otros. En una aplicación industrial por ejemplo, el factor $F_{1}$ puede ser la cantidad de combustible de tipo $A$ que usa la empresa, $F_{2}$ la cantidad de combustible de tipo $\mathrm{B}, \mathrm{F}_{3}$ la electricidad usada, $\mathrm{F}_{4}$ la cantidad de acero usado en el proceso, $\mathrm{F}_{5}$ la cantidad de plásticos y materiales no degradables que consume la empresa, $F_{6}$ los metros cuadrados construidos, $F_{7}$ la cantidad de personas que trabajan en la empresa, entre otros posibles factores.

Es justamente en los valores de los factores de conversión y equivalencia y cuales variables $F_{i}$ considerar en el cálculo en lo que los métodos difieren y en lo que las instituciones y las empresas discrepan, Esto ha llevado a que no se tenga aún un método aceptable y general para determinar y comparar la HdC productos, servicios, viviendas, y empresas. La solución a estas diferencias y cuestionamientos a la utilidad de la $\mathrm{HdC}$ como indicador de sustentabilidad ambiental, cuando se trata de productos, podría tener alguna solución con la aprobación del estándar ISO 14067 y sus normas ISO 14067-1 Huella de Carbono de Productos, Cuantificación e ISO 14067-2 Huella de Carbono de Productos, Comunicación.

\section{APLICACIONES}

Entre las muchas iniciativas que se han realizado para analizar el impacto real de productos seleccionados en el medio ambiente, para introducir el concepto de HdC y sentar las bases de una normativa más estándar para regular la cuantificación de los GEI usando este concepto de $\mathrm{HdC}$ es el proyecto Product Carbon Footprint desarrollado en Alemania durante varios años y del que se informó sus resultados el año 2009 (www.pcf-projekt.de). El método fue el análisis del ciclo de vida de los productos usando la norma ISO 14040/44. El proyecto establece varias conclusiones importantes que es necesario considerar en un estudio futuro y en una propuesta de estandarización de la metodología para determinar la HdC. El informe establece por ejemplo que la norma ISO 14040/44 provee una base sólida para calcular la HdC de bienes y servicios.

En el ámbito más doméstico, existe en Internet una serie de calculadoras de la HdC de una vivienda y su familia, algunas de dudosa validez, pero están allí; y a veces los usuarios (alumnos por ejemplo) hacen uso de ellas sin saber de sus alcances y exactitud. Paul Rauber editor de la revista Sierra ha publicado un interesante estudio sobre calculadoras disponibles en la Web para estimar la HdC (Rauber, 2008). Entre éstas, el autor considera las de tipo educacional, de gobierno, de organizaciones no-gubernamentales, y de tipo comercial, La mayoría de las calculadoras expresan la $\mathrm{HdC}$ en toneladas por año de $\mathrm{CO}_{2}$ equivalente, aunque algunas lo expresan en terreno equivalente que seria necesario tener para absorber las emisiones de gases. Rauber (2008) establece que algunas calculadoras son de dudosa calidad y de exactitud muy incierta. De las nueve calculadoras evaluadas por el autor, se obtuvo para la misma aplicación valores que van desde 8.8 hasta 34 toneladas de $\mathrm{CO}_{2}$ por año. Un resumen del estudio se presenta en la Tabla 2.

Algunas calculadoras enfatizan los gastos de energía, automóvil y transporte aéreo y la mayoría también realiza los cálculos con información promedio que el usuario provee sobre sus usos de energía y eliminación de desechos. Algunos calculadores usan en su metodología promedios regionales o mundiales, asunto que a juicio del autor no parece razonable considerando la gran diferencia en emisiones en distintas regiones y distintos países en el mundo. En Estados Unidos, por ejemplo, el promedio de emisiones por habitante es de 19 toneladas de $\mathrm{CO}_{2}$, en Alemania es 10 toneladas, en México es 4.1, y en Haití es 0.2, siendo el promedio mundial de 6.0 toneladas por habitante (Carbon Planet, 2011). 
Tabla 2: Comparación de algunas calculadoras de la HdC disponibles en la Web. Los valores en la columna $\mathrm{R}$ de Resultados están en toneladas métricas de $\mathrm{CO}_{2}$ por año. $\mathrm{GR}$ en la penúltima columna es Guarda Resultados. Adaptada de Rauber (2008)

\begin{tabular}{|c|c|c|c|c|c|}
\hline Organización & Alcance & Dificultad y Transparencia & Destacable & GR & $\mathbf{R}$ \\
\hline $\begin{array}{l}\text { Berkeley Institute } \\
\text { of the Environment } \\
\text { (educacional) }\end{array}$ & $\begin{array}{l}\text { Amplio: Casa, } \\
\text { transporte, alimentos, } \\
\text { servicios, y emisiones } \\
\text { indirectas. }\end{array}$ & $\begin{array}{l}\text { Muy compleja, requiere muchas } \\
\text { variables incluyendo información } \\
\text { indirecta. Muchos cálculos. }\end{array}$ & $\begin{array}{l}\text { Buen resumen de } \\
\text { resultados en forma } \\
\text { gráfica. }\end{array}$ & Si & 11.1 \\
\hline $\begin{array}{l}\text { British Petroleum, } \\
\text { BP (comercial) }\end{array}$ & $\begin{array}{l}\text { Modesto: casa y } \\
\text { transporte, } \\
\text { incluyendo viajes de } \\
\text { negocios }\end{array}$ & $\begin{array}{l}\text { Baja dificultad, muchos valores } \\
\text { promedios. No dice como se } \\
\text { hacen los cálculos. }\end{array}$ & $\begin{array}{l}\text { Destacable que una } \\
\text { gran empresa de } \\
\text { petróleo se preocupe } \\
\text { del tema }\end{array}$ & No & 19 \\
\hline $\begin{array}{l}\text { EPA } \\
\text { (gubernamental/ } \\
\text { educacional) }\end{array}$ & $\begin{array}{l}\text { Modesto: casa y } \\
\text { transporte, y algo } \\
\text { sobre el estilo de vida }\end{array}$ & $\begin{array}{l}\text { Dificultad media, con una planilla } \\
\text { Excel manejable y con los } \\
\text { resultados a la vista. }\end{array}$ & $\begin{array}{l}\text { Muchos enlaces a } \\
\text { otros calculadores } \\
\text { mas especializados }\end{array}$ & No & 9.69 \\
\hline $\begin{array}{l}\text { Global Footprint } \\
\text { Network (ONG/ } \\
\text { educacional) }\end{array}$ & $\begin{array}{l}\text { Amplio: Casa, } \\
\text { transporte, alimentos, } \\
\text { consumos y reciclaje }\end{array}$ & $\begin{array}{l}\text { Alta dificultad, y va más allá de la } \\
\text { huella del carbono determinando } \\
\text { otros impactos ambientales en el } \\
\text { estilo de vida }\end{array}$ & $\begin{array}{l}\text { Animación molesta } \\
\text { pero otorga mayor } \\
\text { información sobre el } \\
\text { impacto de la huella }\end{array}$ & Sí & 19.6 \\
\hline $\begin{array}{l}\text { An Inconvenient } \\
\text { Truth/ Native } \\
\text { Energy (comercial/ } \\
\text { vende servicios) }\end{array}$ & $\begin{array}{l}\text { Limitado: Casa y } \\
\text { transporte solamente }\end{array}$ & $\begin{array}{l}\text { Baja dificultad estima gastos } \\
\text { energéticos por la cuenta que se } \\
\text { paga. Las instrucciones no son } \\
\text { muy claras }\end{array}$ & $\begin{array}{l}\text { Usa diferentes } \\
\text { factores de emisión } \\
\text { en particular para } \\
\text { transporte. }\end{array}$ & No & 8.85 \\
\hline $\begin{array}{l}\text { Low Impact Living } \\
\text { (comercial/ vende } \\
\text { artefactos) }\end{array}$ & $\begin{array}{l}\text { Muy amplio: Casa, } \\
\text { transporte, artefactos, } \\
\text { basura y agua. }\end{array}$ & $\begin{array}{l}\text { Alta dificultad. Indica estar basada } \\
\text { en literatura de alto nivel pero no } \\
\text { se dan detalles }\end{array}$ & $\begin{array}{l}\text { Entrega información } \\
\text { del dinero perdido y } \\
\text { los ahorros por } \\
\text { acciones favorables }\end{array}$ & Sí & 11.6 \\
\hline $\begin{array}{l}\text { Nature } \\
\text { Conservancy } \\
\text { (ONG/ } \\
\text { educacional/ } \\
\text { vende servicios) }\end{array}$ & $\begin{array}{l}\text { Amplio: Casa, } \\
\text { transporte, alimento y } \\
\text { reciclaje }\end{array}$ & $\begin{array}{l}\text { Baja dificultad con muchos } \\
\text { valores medios e incluye } \\
\text { emisiones indirectas. Las } \\
\text { instrucciones no son claras }\end{array}$ & $\begin{array}{l}\text { Buenos gráficos que } \\
\text { comparan resultados } \\
\text { con promedios en } \\
\text { USA y mundiales }\end{array}$ & No & 25.35 \\
\hline $\begin{array}{l}\text { Redefining } \\
\text { Progress } \\
\text { (ONG/ } \\
\text { educacional/ } \\
\text { vende servicios) }\end{array}$ & $\begin{array}{l}\text { Muy amplio: Casa, } \\
\text { transporte, alimentos, } \\
\text { agua, reciclaje y } \\
\text { consumos }\end{array}$ & $\begin{array}{l}\text { Alta dificultad con cálculos en } \\
\text { rangos y va más allá de las } \\
\text { emisiones de carbono. }\end{array}$ & $\begin{array}{l}\text { Cuantifica el } \\
\text { consumo de energía } \\
\text { en forma detallada }\end{array}$ & No & 63.1 \\
\hline $\begin{array}{l}\text { Terrapass } \\
\text { (comercial/ vende } \\
\text { servicios) }\end{array}$ & $\begin{array}{l}\text { Limitado; casa y } \\
\text { transporte }\end{array}$ & $\begin{array}{l}\text { Dificultad media y enfocada a } \\
\text { transporte en avión. Es confusa } \\
\text { sin explicaciones }\end{array}$ & $\begin{array}{l}\text { Calcula consumos y } \\
\text { permite comparar su } \\
\text { consumo con otras } \\
\text { casas del área }\end{array}$ & No & 9.8 \\
\hline
\end{tabular}

También, algunas calculadoras son muy detalladas y pueden requerir esfuerzo adicional para reunir los datos necesarios para los cálculos. Algunas requieren las cuentas de electricidad, la cantidad y tipo de ampolletas (bombillos) que usa en casa, los viajes en avión y las distancias recorridas, el tipo de ventanas, los tipos de energía y en que proporción es usada en la casa. Varias de las calculadoras están dirigidas a vender al usuario métodos para mejorar su HdC. Las calculadoras disponibles en Internet están dirigidas al usuario final y a determinar cómo cada individuo aporta a la generación de GEI y a la HdC. No aparece indicado en ninguna calculadora la huella dejada en la manufactura del auto, o en el trasporte del hierro y del acero que fue necesario transportar y usar para construir el auto. Algunas calculadoras pretenden incluir estos factores, pero los valores cambian en forma dramática, no pudiéndose establecer la veracidad de los resultados. Rauber, por ejemplo, obtiene 11.1 toneladas de $\mathrm{CO}_{2}$ con una calculadora, sin incluir gastos de manufactura y 34 incluyéndolos. La verdadera cantidad de emisiones queda en duda. La evaluación de este tipo de calculadoras es un buen tema par discutir en cursos técnicos o universitarios, para trabajos semestrales o incluso para tema de Tesis de grado. 


\section{TRABAJOS QUE PUEDEN DESARROLLAR LOS ALUMNOS}

Los autores han realizado tareas concretas para expandir el conocimiento académico y social del tema. Se ha incorporado el tema en cursos, se han impartido conferencias académicas y de extensión, se han realizado trabajos de tesis y se ha participado en congresos sobre el tema. En el contexto de este trabajo se ha enfatizado siempre la importancia de introducir el concepto en cursos relacionados. La Tabla 3 resume algunas actividades realizadas por nuestro grupo de trabajo en el último tiempo en la difusión de este importante concepto y en la concienciación de diversos grupos.

Tabla 3: Algunas actividades realizadas por los autores en la difusión y enseñanza de la HdC

\begin{tabular}{|c|c|}
\hline Actividad & Título \\
\hline Cursos & $\begin{array}{l}\text { - Métodos de Gestión Avanzada } \\
\text { - Innovación para la competitividad } \\
\text { - Literatura Científica para Ingeniería. La Huella del Carbono }\end{array}$ \\
\hline Tesis dirigidas & $\begin{array}{l}\text { Huella de Carbono para productos exportables del Sector Pectinicultor en Chile: magnitud, } \\
\text { complejidades metodológicas y equivalencias } \\
\text { Euantificación de la Huella De Carbono para el Proceso de Solarización Modificada de la } \\
\text { Empresa Capel Ltda., Como Base para su Incorporación al Mercado de Carbono. } \\
\text { Formulación del Proyecto de Implementación de Estándar en Contabilidad Corporativa para } \\
\text { Reporte de Gases de Efecto Invernadero en la Actividad Acuícola Extensiva. }\end{array}$ \\
\hline Extensión & $\begin{array}{l}\text { - Participación en el Comité Espejo Nacional ISO TC } 207 \text { SC 7, que estudia las normas ISO } \\
\text { 14067-1 Carbon footprint of products } \\
\text { - Participación en el programa de fortalecimiento de capacidades "Gestión Ambiental, temas } \\
\text { emergentes y producción limpia para el desarrollo de nuevos APL" }\end{array}$ \\
\hline $\begin{array}{l}\text { Conferencias y } \\
\text { Congresos }\end{array}$ & $\begin{array}{l}\text { - La Huella del Carbono. Conceptos y Definiciones } \\
\text { - Competitividad y Huella de Carbono } \\
\text { - Desde la Huella Ecológica a la Huella de Carbono } \\
\text { - Análisis y Evaluación de Calculadoras de la Huella de Carbono }\end{array}$ \\
\hline Publicaciones & $\begin{array}{l}\text { Huella de Carbono. Parte 1: Conceptos, Métodos de Estimación y Complejidades } \\
\text { Metodológicas } \\
\text { - Huella de Carbono. Parte 2: Las Visión de las Empresas, los Cuestionamientos y el Futuro }\end{array}$ \\
\hline
\end{tabular}

Una forma práctica y que tiene buena aceptación por parte de los estudiantes es el trabajo semestral guiado ya sea individual o en grupos sobre un tema definido por el profesor y en lo posible de interés para el alumno, dependiendo de la especialidad que esté cursando. Por ejemplo si se trata de alumnos de ingenieria industrial probablemente temas relacionados con modelos para cuantificar la $\mathrm{HdC}$ o para gestionar recursos relacionados con ella, sean de especial interés. Si se trata de alumnos de ingenieria en minas, el tema de la $\mathrm{HdC}$ en la industria del cobre, en la minería no-metálica u otras similares, seguro que entusiasmarán a los estudiantes. Para un alumno de ingeniería química o de química industrial, estudiar la importancia de los gases usualmente considerados en la cuantificación de la huella del carbono (y porqué no se consideran otros igualmente dañinos), puede ser de especial atractivo. Estudiar y evaluar la $\mathrm{HdC}$ en vertederos de residuos sólidos por ejemplo debe ser muy motivador para un estudiante de ingeniería ambiental. La Tabla 3 muestra una selección de los muchos temas que pueden ser tratados en cursos directa o indirectamente relacionados con medio ambiente.

El trabajo semestral permite también practicar otras áreas de especial importancia en la formación de profesionales: búsqueda eficiente y eficaz de la literatura, lectura de artículos de revistas de corriente principal, selección de material adecuado y pertinente, redacción de informes técnicos, y exposición frente a sus compañeros.

\section{CONCLUSIONES}

La revisión de la literatura, aunque no fue necesariamente exhaustiva, muestra que el tema está llegando de a poco a los usuarios y a quienes provocan nuestros problemas ambientales (nosotros mismos). 
Los alumnos muestran interés por el tema y se convencen de su importancia cuando realizan sus trabajos de investigación, más que cuando el instructor les relata las materias en clases.

Los trabajos preliminares en cursos y trabajos semestrales y en temas de tesis de pregrado y posgrado han resultado positivos para ir inculcando el concepto en los alumnos y futuros profesionales.

El estudio realizado hasta la fecha justifica su inclusión en cursos de ingeniera y ciencias y muestra que nuestros futuros profesionales deben llevar las bases mínimas que les permitan entender el concepto y aplicarlo durante su vida profesional.

Tabla 3: Selección de temas sobre la HdC que pueden ser asignados en un curso de pregrado

\begin{tabular}{|c|c|c|}
\hline$N^{\circ}$ & Tema & Detalle \\
\hline 1 & Conceptos y definiciones de la $\mathrm{HdC}$ & $\begin{array}{l}\text { Revisión crítica de la literatura sobre los distintos enfoques } \\
\text { académicos y empresariales }\end{array}$ \\
\hline 2 & $\begin{array}{l}\text { Huella del carbono y cambio climático } \\
\text { global }\end{array}$ & $\begin{array}{l}\text { Relación conceptual entre HdC y cambio climático, y conceptos } \\
\text { erróneos sobre el tema }\end{array}$ \\
\hline 3 & Huella del carbono y globalización & $\begin{array}{l}\text { La influencia del concepto de emisiones y HdC en la globalización } \\
\text { industrial }\end{array}$ \\
\hline 4 & Huella del carbono y la industria & $\begin{array}{l}\text { Definir las distintas industrias o áreas. Por ejemplo la industria } \\
\text { turística, de alimentos, automotriz, agricultura }\end{array}$ \\
\hline 5 & $\begin{array}{l}\text { Huella del carbono en catástrofes } \\
\text { naturales }\end{array}$ & Terremotos, maremotos, sequías, inundaciones, huracanes \\
\hline 6 & $\begin{array}{l}\text { Huella del carbono en incendios } \\
\text { forestales }\end{array}$ & Estudiar los incendios intencionales o por descuido humano \\
\hline 7 & Huella del carbono en el transporte & $\begin{array}{l}\text { Se puede dividir en transporte terrestre (camiones y trenes), } \\
\text { marítimo y aéreo }\end{array}$ \\
\hline 8 & $\begin{array}{l}\text { Huella del carbono en derrames de } \\
\text { petróleo }\end{array}$ & $\begin{array}{l}\text { Efectos de los derrames causados por embarcaciones o fallas en } \\
\text { pozos de alta mar }\end{array}$ \\
\hline 9 & $\begin{array}{l}\text { Huella del carbono en vertederos de } \\
\text { residuos }\end{array}$ & Se pueden incluir vertederos y plantas de tratamientos de residuos \\
\hline 10 & Huella del carbono en el turismo & $\begin{array}{l}\text { Considerar actividades en la playa y campo, ciudades, transporte, } \\
\text { recorridos por la ciudad }\end{array}$ \\
\hline 11 & Huella del carbono en la agricultura & $\begin{array}{l}\text { Considerar pesticidas y otros agroquímicos, como también los } \\
\text { residuos de cosecha }\end{array}$ \\
\hline 12 & Modelos para cuantificar la $\mathrm{HdC}$ & $\begin{array}{l}\text { Identificar modelos, clasificarlos y compararlos por simplicidad y } \\
\text { exactitud }\end{array}$ \\
\hline 13 & $\begin{array}{l}\text { Huella del carbono en la industria } \\
\text { vínica }\end{array}$ & $\begin{array}{l}\text { Considerar vinos y licores, incluyendo destilación alcohólica y } \\
\text { transporte }\end{array}$ \\
\hline
\end{tabular}

\section{AGRADECIMIENTOS}

Los autores agradecen el apoyo de la Universidad de La Serena y del Centro de Información Tecnológica (Chile) para la realización de este trabajo. CE agradece también al Depto. Adm. Empresas y Gestión Económica de Rec. Naturales, de la Universidad de Lleida (España).

\section{REFERENCIAS}

BC, Bilan Carbone http://www2.ademe.fr/servlet/KBaseShow?sort=-1ycid=96ym=3ycatid=15730 (2011)

Boiral, O. Corporate Response to Global Warming: For a Proactive Strategy , Int. J. Business and Economics Perspectives, 1(1), 79-95 (2006)

BSI, Bristish Standards Institute, PAS 2050: 2008. Specification for the assessment of the life cycle greenhouse emissions of goods and services, http://www.bsigroup.com/en/Standards-andPublications/Industry-Sectors/Energy/PAS-2050/ (2008) 
Carballo, A. y García, M. C., La huella ecológica y su aplicación a organizaciones: él caso de una empresa conservera en Galicia, España. DELOS, 1, 1-18. (2008)

Carbon Planet, http://www.carbonplanet.com/country_emissions (2011)

Clément C. y Lenne P., Metodologías de cálculo de la Huella de Carbono y sus potenciales implicaciones para América Latina, documentos de trabajo, Comisión Económica para América Latina y el Caribe (2010).

CT, Carbon footprint measuring methodology, The Carbon Trust, Londres, UK (2007)

De La Torre, A., Fajnzylber, P. y Nash, J., Desarrollo con menos carbono: respuestas latinoamericanas al desafío del cambio climático. Banco Central, Washington D.C., USA (2009)

Doménech, J.L., Huella ecológica portuaria y desarrollo sostenible. Puertos 114, 26-31 (2004a)

Doménech, J.L. La huella ecológica empresarial: el caso del puerto de Gijón. Working paper presented at the VII National conference of environment, Madrid, Spain, November (2004b)

Doménech, J.L., Huella ecológica y desarrollo sostenible. Aenor Ediciones, Madrid-España (2007)

EPA, United States Environmental Protection Agency, www.epa.gov (2007)

Protocolo GEI Initiative. The Protocolo GEI for project accounting. http://www.ghgprotocol.org/files/ ghg_project_protocol.pdf (2005)

IPCC, Intergovernmental Panel on Climate Change, http://www.ipcc.ch/ (2007)

Minx, J. C., Wiedmann, T. y otros 14 autores, Input-output analysis and carbon footprinting: an overview of applications", Economic Systems Research, 21:3,187-216 (2010)

NEA, National Education Association, Teaching Students about Carbon Emissions, http://www.nea.org/tools/14841.htm (2011)

Peters G.P. y E.G. Hertwich, CO2 Embodied in International Trade with Implications for Global Climate Policy, Environ. Sci. Technol., 2008, 42 (5), pp 1401-1407, 30, 2008

Plasmann, K.; Norton, A.; y otros 4 autores, Methodological complexities of product carbon footprinting: a sensitivity analysis of key variables in a developing country context, Env. Sci. y Police, 13,393-404 (2010)

Rauber, P., Carbon Confessional. How to come clean about your greenhouse gasses, revista Sierra, http://www.sierraclub.org/sierra/200809/carbon.asp (2008)

Schneider, H. y Samaniego, J.L., La huella del carbono en la producción, distribución y consumo de bienes y servicios, documentos de proyectos, $N^{\circ} 298$, Santiago de Chile, Comisión Económica para América Latina y el Caribe, CEPAL (2009)

SchoolCoop, Teaching Materials, http://teacher.beecoop.co.uk/?q=node/246 (2011)

Wackernag, M. y W.E. Rees, Perceptual and structural barriers to investing in natural capital: Economics from an ecological footprint perspective, Ecological Economics, 20(1), 3-24 (1997)

Wiedmann, T. y J. Minx, A Definition of Carbon Footprint In: C. C. Pertsova, Ecological Economics Research Trends: Chapter 1, pp. 1-11, Nova Science Publishers, Hauppauge NY, USA. https://www.novapublishers.com/catalog/product_info.php?products_id=5999 (2008)

Wittneben, B., Climate change basics for managers. Manag. Decision, 47, 1122-1132 (2009)

Wittneben, B y Kiyar, D., Climate change basics for managers, Management Decision, 47(7), 1122-1132 (2009)

WRI, World Resources Institute, Washington, DC, USA, www.wri.org 